\title{
Editorial \\ Stroke in the Young 2012
}

\author{
Halvor Naess, ${ }^{1}$ Turgut Tatlisumak, ${ }^{2}$ and Janika Kõrv ${ }^{3}$ \\ ${ }^{1}$ Department of Neurology, Haukeland University Hospital, N-5021 Bergen, Norway \\ ${ }^{2}$ University of Helsinki, 00290 Helsinki, Finland \\ ${ }^{3}$ Department of Neurology and Neurosurgery, University of Tartu, 51014 Tartu, Estonia
}

Correspondence should be addressed to Halvor Naess, haln@haukeland.no

Received 2 July 2012; Accepted 2 July 2012

Copyright () 2012 Halvor Naess et al. This is an open access article distributed under the Creative Commons Attribution License, which permits unrestricted use, distribution, and reproduction in any medium, provided the original work is properly cited.

This is the second special issue on stroke in the young. In recent years there has been an increasing research activity concerning stroke in young adults and children. Important data has been published as to long-term outcome in young adults with cerebral infarction. Long-term mortality is high compared to controls without stroke and more is known about risk factors both regarding mortality and recurrence of stroke or other vascular events. This knowledge helps physicians to target secondary preventive medication. Most prognostic studies have been performed among young patients with cerebral infarction. Less is known about prognosis after cerebral hemorrhage. This is at least partly due to lower frequency of cerebral hemorrhage than cerebral infarction. We hope that more research will be performed among young patients with cerebral hemorrhage in the future. In addition, more studies from developing countries and from nonwhite populations are needed in all aspects of ischemic and hemorrhagic stroke in the young and in children.

The range of causes underlying stroke is wider in children and young adults than in old stroke patients. This makes diagnosis and choice of treatment particularly challenging in young patients. The present issue includes 6 papers which offer a small contribution to the knowledge that may help physicians reach better decisions as to providing better care for young stroke patients.

This special issue includes 6 articles ranging from perinatal stroke to long-term survival after stroke in young adults. One paper reports factors associated with social, thought, and attention problems in children with perinatal stroke. These factors included male gender, neonatal seizures, and uteroplacental insufficiency. A study from China compares the distribution of risk factors in young stroke patients with old stroke patients. Hypertension and diabetes mellitus were equally frequent among young and old stroke patients, whereas smoking and heavy drinking were much more frequent among young stroke patients. Heart disease was more common among old stroke patients. A study from Canada shows that ischemic stroke is associated with acute exposure to ambient sulfur dioxide. A comparison of provision of stroke care in younger and older patients in London shows that younger patients had equal or greater access to evidence-based care than older patients. A study from Sweden including more than 10,000 stroke patients, 40-59 years old, shows that low income was associated with higher long-term mortality. A study from Estonia compares long-term survival of young stroke patients included in two time periods (1991-1993 and 2001-2003). Patients with intracerebral hemorrhage had the lowest survival. Five-year survival rate for patients with ischemic stroke 15 to 44 years was $75 \%$. There was no difference in overall long-term survival for the two time periods.

We hope this special issue presents information that may be of help in caring for children and young adults with stroke and that it will unleash ideas for future research.

Halvor Naess Turgut Tatlisumak Janika Kõrv 


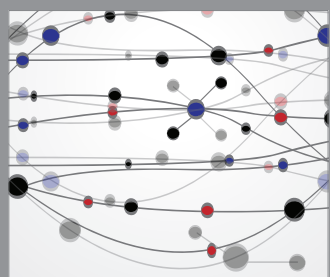

The Scientific World Journal
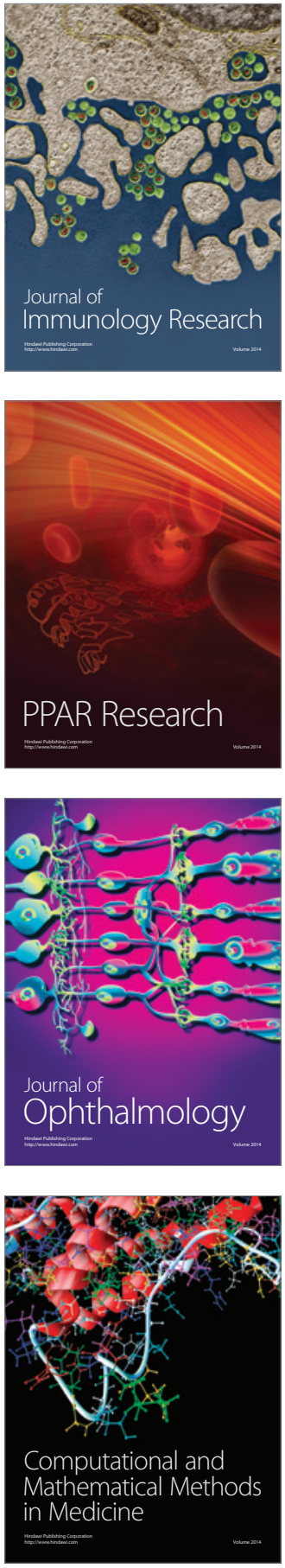

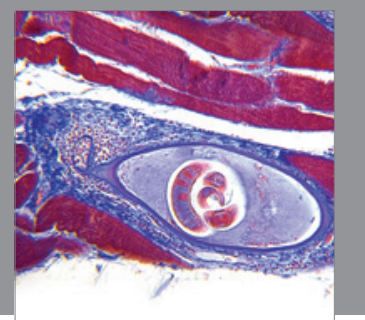

Gastroenterology

Research and Practice
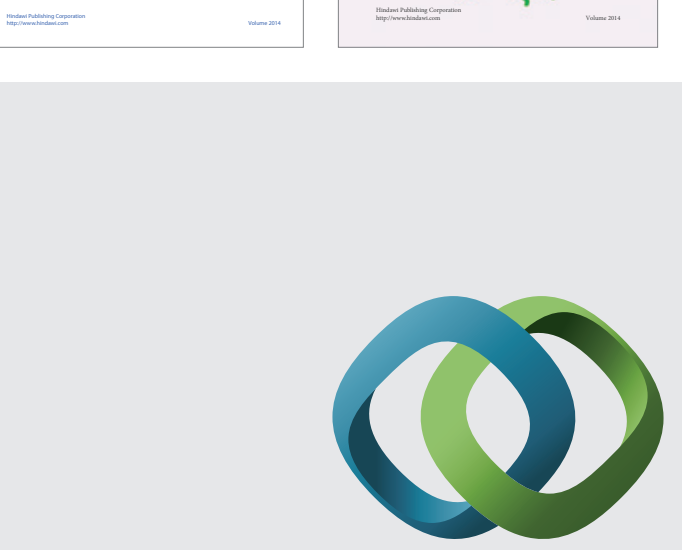

\section{Hindawi}

Submit your manuscripts at

http://www.hindawi.com
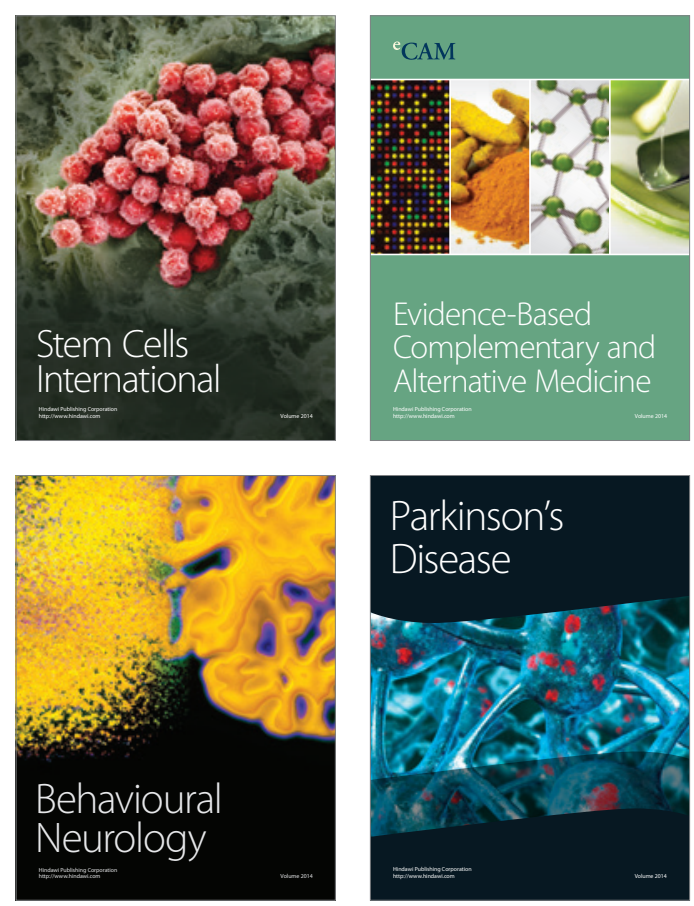

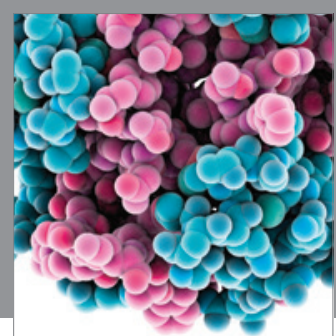

Journal of
Diabetes Research

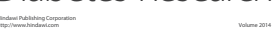

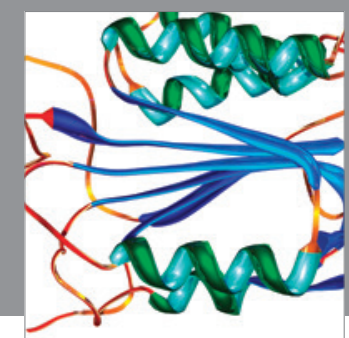

Disease Markers
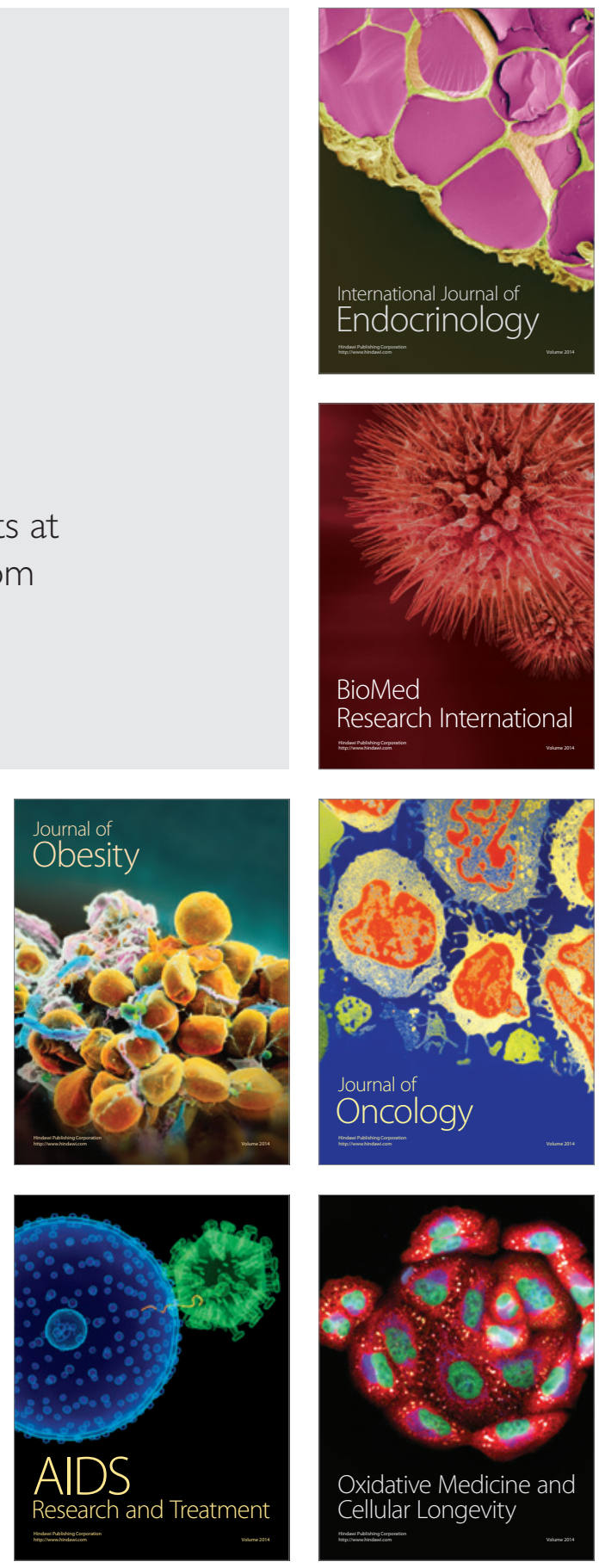